\title{
CHAIN THEORIES AND THEIR DERIVED HOMOLOGY
}

\author{
R. O. BURDICK, P. E. CONNER AND E. E. FLOYD
}

In this note we shall prove that the generalized homology theory known as oriented bordism [1] cannot be derived from an underlying chain theory. This will follow from our main theorem which characterizes those generalized homologies which arise from chain theories.

Consider a covariant functor $L$ from the category of finite $C W$-pairs to the category of chain complexes. For each finite $\mathrm{CW}$-pair $(X, A)$ we thus have the chain complex

$$
L(X, A): \cdots \stackrel{\partial}{\leftarrow} L_{n-1}(X, A) \stackrel{\partial}{\leftarrow} L_{n}(X, A) \leftarrow \cdots
$$

and for each map $f:(X, A) \rightarrow(Y, B)$ we have a chain map $L(f): L(X, A)$ $\rightarrow L(Y, B)$ of degree 0 . We denote $L(f)$ by $f_{\sharp}$; also $L(X, \varnothing)$ is denoted by $L(X)$. We call $L$ a chain theory if

(1) for each finite $\mathrm{CW}$-pair $(X, A)$ the sequences

$$
0 \rightarrow L_{n}(A) \rightarrow L_{n}(X) \rightarrow L_{n}(X, A) \rightarrow 0
$$

are all exact,

(2) the derived homology functor is a generalized homology theory.

By (2) we mean that the derived homology functor satisfies the Eilenberg-Steenrod axioms except possibly for the dimension axiom. We now state our principal result.

ThEOREM. Let $L$ be a chain theory and set $h_{n}(X, A)=H_{n}(L(X, A)), h_{n}$ $=h_{n}(\mathrm{pt})$. Then for each finite CW-pair $(X, A)$,

$$
h_{n}(X, A) \simeq \sum_{p+q=n} H_{p}\left(X, A ; h_{q}\right)
$$

where $H_{p}\left(X, A ; h_{q}\right)$ denotes singular homology with coefficients $h_{q}$. Furthermore, this isomorphism is a natural equivalence of homology theories.

Thus the derived homology theory is uniquely determined by its coefficient groups $h_{q}(\mathrm{pt})$, and is always trivial. In particular it will follow that oriented bordism cannot be derived from an underlying chain theory.

Our proof is an exercise in the use of acyclic models; for the appropriate definitions we refer to Eilenberg-MacLane [2] and to Spanier [3, p. 165]. We shall need the following variant of acyclic models.

Received by the editors May 11, 1967. 
Definition. A space $M$ is point-like with respect to $L$ if and only if $\epsilon: M \rightarrow$ pt induces an isomorphism $\epsilon_{*}: h_{*}(M) \simeq h_{*}$.

We denote by $\mathfrak{M}$ a collection of point-like models.

Lemma 1. Let $K$ and $L$ be chain theories and $\mathfrak{M}$ a collection of models, point-like with respect to $L$. Suppose that each functor $K_{n}$ is representable by the models $\mathfrak{M}$ and that there is an integer $N$ with $K_{n}=0$ for all $n<N$, then any chain map $f: K(\mathrm{pt}) \rightarrow L(\mathrm{pt})$ can be extended to a natural transformation of $K \rightarrow L$, assigning to each $(X, A)$ a chain map $F: K(X, A)$ $\rightarrow L(X, A)$ of degree 0 .

Suppose for each $(X, A)$ and for each $p$ with $p<n$ there have been defined homomorphisms $F: K_{p}(X, A) \rightarrow L_{p}(X, A)$ such that

(1) $\partial F=F \partial$ on each $K_{p}(X, A)$,

(2) if $\phi:(X, A) \rightarrow(Y, B)$ is a map then $\phi_{f} F=F \phi_{f}$,

(3) $F=f$ on $K_{p}(\mathrm{pt})$.

We must define $F$ on each $K_{n}(X, A)$; first we define $F$ on $K_{n}(M)$ for $M \in \mathfrak{M}$.

For each $M \in \mathfrak{M}$ select a base point $x_{0} \in M$ and let $K_{q}(M)^{-}$denote the kernel of $\epsilon_{\sharp}: K_{q}(M) \rightarrow K_{q}\left(x_{0}\right)$; similarly define $L_{q}(M)^{-}$. In view of (2) above, $F\left(K_{p}(M)\right)^{-} \subset L_{p}(M)^{-}$for $p<n$. There is the diagram

$$
\begin{aligned}
& K_{n}(M)^{-} \stackrel{\partial}{\rightarrow} K_{n-1}(M)^{-} \stackrel{\partial}{\rightarrow} K_{n-2}(M)^{-} \\
& \downarrow F \quad \downarrow F \\
& L_{n}(M)^{\sim} \stackrel{\partial}{\rightarrow} L_{n-1}(M)^{-} \stackrel{\partial}{\rightarrow} L_{n-2}(M)^{-} .
\end{aligned}
$$

Since $M$ is point-like with respect to $K$, then $H_{n-1}(L(M))^{-}=0$ and the bottom row is exact. Hence

$$
\operatorname{Im}\left(F \partial: K_{n}(M)^{-} \rightarrow L_{n-1}(M)\right)^{\mathcal{}} \subset \operatorname{Im}\left(\partial: L_{n}(M)^{\mathcal{}} \rightarrow L_{n-1}(M)\right)^{\mathcal{}} .
$$

Since $K_{n}$ is representable, then $K_{n}(M)$ and also $K_{n}(M)^{-}$are free, thus there exists a homomorphism $F: K_{n}(M)^{-} \rightarrow L_{n}(M)^{-}$so that commutativity holds in the above diagram.

Note that we may consider $F: K_{n}\left(x_{0}\right) \rightarrow L_{n}\left(x_{0}\right)$ as given since $F=f$. We shall obtain splittings

$$
K(M)=K(M)^{\sim} \oplus K\left(x_{0}\right), \quad L(M)=L(M)^{\sim} \oplus L\left(x_{0}\right)
$$

and we define $F: K(M)^{\sim} \rightarrow L(M)^{-}$as the direct sum of

$$
F: K_{n}(M)^{\sim} \rightarrow L_{n}(M)^{-}, \quad F: K_{n}\left(x_{0}\right) \rightarrow L_{n}\left(x_{0}\right) .
$$

The splittings are obtained by considering $i: x_{0} \rightarrow M$, identifying $i_{f}\left(K\left(x_{0}\right)\right)$ with $K\left(x_{0}\right)$. We thus obtain $F: K_{n}(M) \rightarrow L_{n}(M)$ with $F \partial=\partial F$. 
We extend $F$ to all $K_{n}(X)$ in the following standard way. Let $K_{n}(X) \wedge$ be the free abelian group generated by all pairs $(\phi, m)$ where $\phi: M \rightarrow X$ is a map of a model into $X$ and $m \in K_{n}(M)$. There is the natural transformation $\Phi: K_{n}(X)^{\wedge} \rightarrow K_{n}(X)$ given by $(\phi, m) \rightarrow \phi_{\sharp}(m)$. By the assumption of representability there is a $\Psi: K_{n}(X) \rightarrow K_{n}(X)^{\wedge}$, which is natural, with $\Phi \Psi=$ identity. We define $F^{\wedge}: K_{n}(X)^{\wedge} \rightarrow L_{n}(X)$ by

$$
F(\phi, m)^{\wedge}=\phi \sharp F(m),
$$

$F: K_{n}(X) \rightarrow L_{n}(X)$ by $F^{\wedge} \Psi$. The reader may show that $\partial F^{\wedge}=F \partial \Phi$.

To obtain $F: K_{n}(X, A) \rightarrow L_{n}(X, A)$ we simply complete the diagram

$$
\begin{aligned}
& 0 \rightarrow K_{n}(A) \rightarrow K_{n}(X) \rightarrow K_{n}(X, A) \rightarrow 0 \\
& F \downarrow \quad F \downarrow \quad \downarrow \\
& 0 \rightarrow L_{n}(A) \rightarrow L_{n}(X) \rightarrow L_{n}(X, A) \rightarrow 0
\end{aligned}
$$

By induction over $n$, Lemma 1 is thus proved.

Lemma 2. Fix a chain theory $L$ and an integer $n$, then there exist natural transformations

$$
H_{m}\left(X, A ; h_{n}\right) \rightarrow h_{m+n}(X, A)
$$

defined for all pairs $(X, A)$ and all $m$ such that $H_{0}\left(\mathrm{pt} ; h_{n}\right) \simeq h_{n}(\mathrm{pt})$.

Let $S(X, A)$ denote the singular chain complex of $(X, A)$ and recall that each $S_{n}(X)$ is representable with model the standard $n$-simplex. We shall consider a free chain complex $C$ and associate with each pair $(X, A)$ the chain complex $S(X, A) \otimes C$. Then $(S(X) \otimes C)_{n}$ is seen to be representable with models the set of standard simplices. By the homotopy axiom for $h(X)=H(L(X))$, the standard simplex is pointlike with respect to $L$. If there exists an integer $N$ with $C_{n}=0$ for $n<N$, then it follows from Lemma 1 that a given chain map

$$
f: S(\mathrm{pt}) \otimes C \rightarrow L(\mathrm{pt})
$$

can be extended to

$$
F: S(X, A) \otimes C \rightarrow L(X, A) .
$$

We shall now choose the complex $C$. Let the abelian group $h_{n}(\mathrm{pt})$ have free resolution

$$
0 \leftarrow h_{n}(\mathrm{pt}) \leftarrow F_{0} \leftarrow F_{1} \leftarrow 0
$$

and then take $C$ to be the chain complex

$$
C: \cdots \leftarrow 0 \leftarrow\left(C_{n}=F_{0}\right) \leftarrow\left(C_{n+1}=F_{1}\right) \leftarrow 0 \cdots .
$$


Consider now the diagram

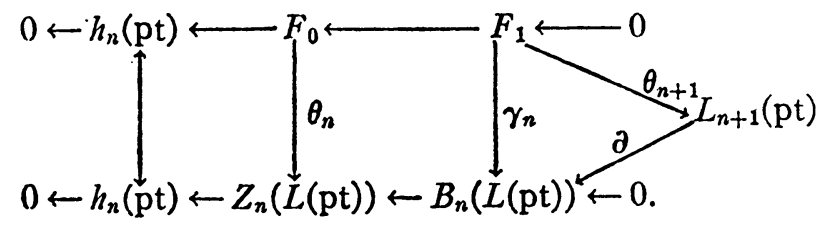

Here $\theta_{n}, \gamma_{n}, \theta_{n+1}$ are chosen so that the diagram commutes. There results a chain map $\theta: C \rightarrow L(\mathrm{pt})$ such that $\theta_{*}: H_{n}(C) \simeq h_{n}(\mathrm{pt})$. Naturally $H_{i}(C)=0, i \neq n$.

We now choose the chain map $f: S(\mathrm{pt}) \otimes C \rightarrow L(\mathrm{pt})$. Recall that $S_{k}(\mathrm{pt})$ is an infinite cyclic group with a preferred generator $e_{k}$ for $k \geqq 0$. Define

$$
\phi: S(\mathrm{pt}) \otimes C \rightarrow C
$$

by $\phi\left(e_{k} \otimes c\right)=0, k>0, \phi\left(e_{0} \otimes c\right)=c$. Then $\phi$ is a chain equivalence. Let

$$
f=\theta \phi: S(\mathrm{pt}) \otimes C \rightarrow L(\mathrm{pt})
$$

then $f_{*}: H_{n}(S(\mathrm{pt}) \otimes C) \simeq H_{n}(C) \simeq h_{n}(\mathrm{pt})$. We need now only verify the well-known fact that

$$
H_{m+n}(S(X, A) \otimes C) \simeq H_{m}\left(X, A ; h_{n}\right) .
$$

This is clear since the derived homology functor of $S(X, A) \otimes C$ has $H_{i}(S(\mathrm{pt}) \otimes C)=0$ for $i \neq n, H_{n}(S(\mathrm{pt}) \otimes C) \simeq h_{n}(\mathrm{pt})$ and the EilenbergSteenrod uniqueness theorem can be applied.

Our main theorem follows easily from Lemma 2. For there exists natural transformations

$$
\sum_{p+q=m} H_{p}\left(X, A ; h_{q}\right) \rightarrow h_{m}(X, A) .
$$

For $X=\mathrm{pt}$ and $A$ empty this is easily seen to be an isomorphism for each $m$. But if a natural transformation of homology theories is an isomorphism on the coefficient groups in each dimension it is then an isomorphism for each finite $\mathrm{CW}$-pair $(X, A)$. The theorem follows.

\section{REFERENCES}

1. P. E. Conner and E. E. Floyd, Differentiable periodic maps, Ergebnisse Tract No. 33, Springer-Verlag, Berlin, 1964. 199.

2. S. Eilenberg and S. MacLane, Acyclic models, Amer. J. Math. 79 (1953), 189-

3. E. H. Spanier, Algebraic topology, McGraw-Hill Series in Higher Mathematics, New York, 1966. 